\title{
24 in 1 - Der Umzug der Campusbibliothek der Freien Universität Berlin
}

Zusammenfassung: Mehr als eine Million Bücher aus 24 Einzelbibliotheken wurden in die neue Campusbibliothek der Freien Universität transportiert und einheitlich nach der Regensburger Verbundklassifikation aufgestellt. Beteiligt waren Technische Abteilung, Fachpacker der beauftragten Speditionen, Bibliotheksmitarbeiterinnen und -mitarbeiter sowie studentische Hilfskräfte - insgesamt mehr als 100 Personen.

\begin{abstract}
More than a million books from 24 branch libraries were transported to the new "Campusbibliothek der FU Berlin" and arranged according to the Regensburg classification system. This involved the Technical Department, transport and forwarding specialists, library staff and student assistants - a total of more than 100 people.
\end{abstract}

Schlüsselwörter: Bibliotheksumzug, Freie Universität Berlin, Campusbibliothek

Keywords: Library Moving, Freie University Berlin, Campus Library

DOI 10.1515/abitech-2015-0020

\section{Einleitung}

1973: 190 Bibliotheken an der Freien Universität Berlin 2001: 73 Bibliotheken an der Freien Universität Berlin 2014: 10 Bibliotheksbereiche an 25 Standorten 2015: 9 Bibliotheksbereiche an 12 Standorten

Einen Konzentrationsprozess und die damit einhergehende Reorganisation sehen wir bei vielen zweischichtigen Bibliothekssystemen. ${ }^{1}$ An der Freien Universität Berlin

1 Wissenschaftsrat: Stellungnahme zur Bibliotheksversorgung an der Freien Universität Berlin, Berlin 1990. Zur Debatte um die Struktur universitärer Bibliotheksysteme: Jochum, Uwe: Kleine Bibliotheksgeschichte. Stuttgart 2007, S. 133-150; und Naumann, Ulrich: Hochschulbibliothekssysteme im Vergleich. Vorlesungsskript einer
(FU) wurden bereits 2005 im Neubau der Philologischen Bibliothek zwölf Institutsbibliotheken zusammengelegt. Ebenfalls 2005 beschloss der Fachbereichsrat der Fakultät für Politik- und Sozialwissenschaften, seine Bibliotheken zusammenzuführen. ${ }^{2}$ Noch im selben Jahr startete auch der Architektur-Wettbewerb für einen Bibliotheksneubau, den das Büro Florian Nagler Architekten GmbH, München, gewann.

Aufhänger für den Neubau mit seiner heterogenen Fächerverteilung war die Idee, Synergieeffekte für die „Kleinen Fächer“ herzustellen. Unter diesem Gesichtspunkt begann das Projekt von Bibliotheksseite 2007 unter Leitung von Dr. Klaus Werner, der maßgeblich für das Bibliotheksbedarfsprogramm verantwortlich war. Außerdem wurden Wissenschaftler aus den Altertumswissenschaften in die ersten Diskussionen über die neue, fächerübergreifende Aufstellungssystematik (Regensburger Verbundklassifikation, RVK) einbezogen. Als Dr. Andrea Tatai das Projekt 2010 übernahm, startete sie eine große Initiative, die in dem Beschluss mündete, eine vollständige Umarbeitung aller 1,2 Millionen Bände nach RVK bereits vor dem Umzug anzustreben. Konsequenz dessen war eine Personaloffensive mit der Einstellung einer ganzen Reihe von Projektkräften. 2012 beschäftigte das Projekt ,24 in 1“ an 20 Standorten 66 Bibliothekare und Magazinmitarbeiter, davon 11 wissenschaftliche Bibliothekare, sowie über 60 studentische Hilfskräfte.

Der Neubau mit Anteilen für Institute, Fachbereichsverwaltung und Bibliothek ist mit der sanierten Erziehungswissenschaftlichen Bibliothek verbunden und bildet seit April 2015 als Zusammenschluss von 24 Bibliotheken aus fünf Fachbereichen gemeinsam die neue Campusbi-

geplanten Lehrveranstaltung am Institut für Bibliotheks- und Informationswissenschaft der Humboldt-Universität zu Berlin. Berlin 2007. http://userpage.fu-berlin.de/unaumann/

Vorlesungsskript_2007.pdf. (28.04.2015).

2 Naumann, Ulrich: Die Universitätsbibliothek und das Bibliothekssystem der Freien Universität Berlin. In: Fünfzig Jahre Universitätsbibliothek der Freien Universität Berlin, hrsg. von Ulrich Naumann, Doris Fouquet-Plümacher. Berlin 2002, S. 463-519, hier S. 470f. 


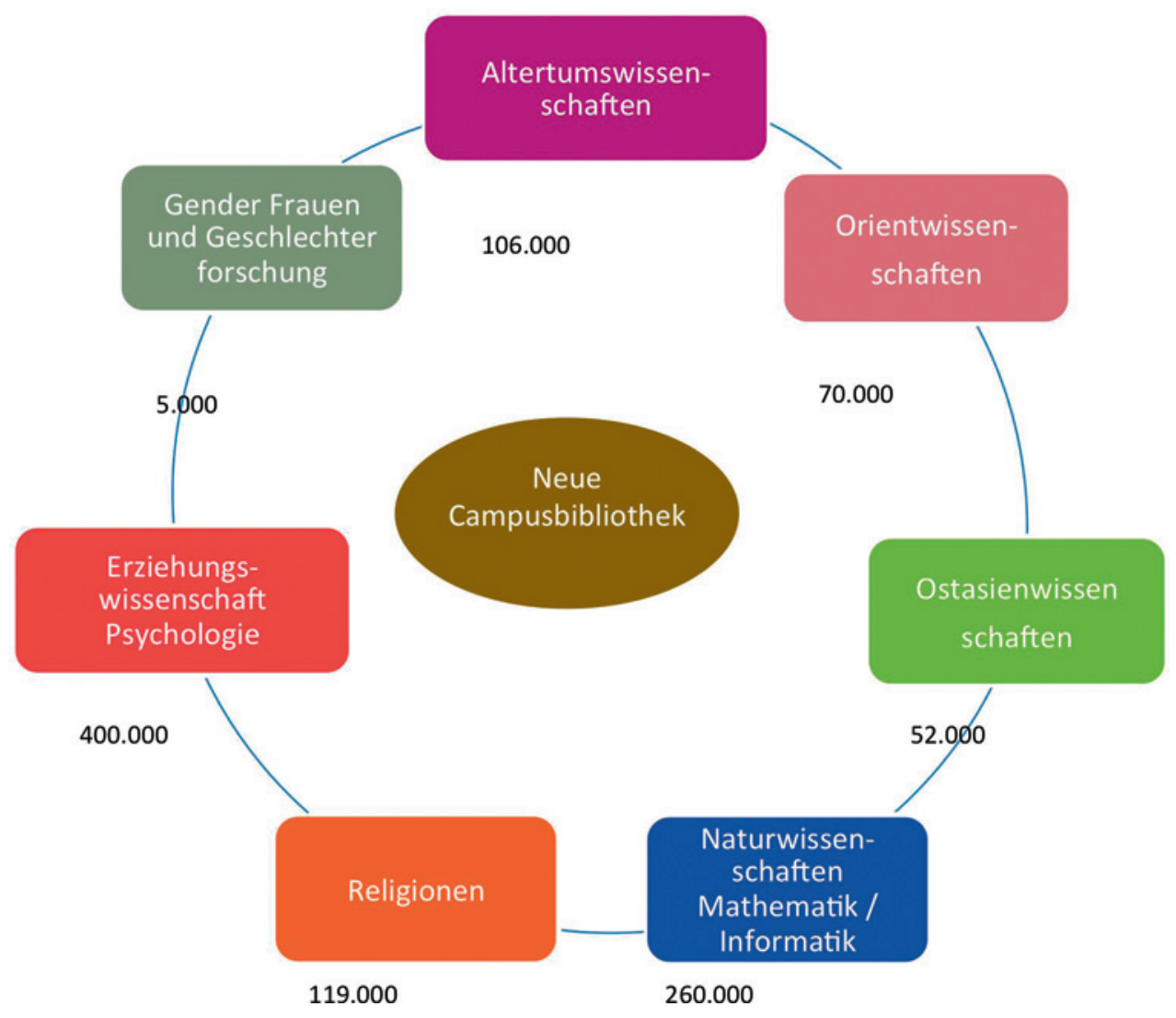

Abb. 1: Übersicht der Fächercluster

bliothek Natur-, Kultur- und Bildungswissenschaften, Mathematik, Informatik, Psychologie.

Der Neubau ist in zwei Bereiche unterteilt: Der zur Fabeckstraße gelegene Gebäudeteil beherbergt über 220 Büros und Arbeitsräume, zwölf Seminarräume und drei Hörsäle für die Mitglieder der Institute aus dem Fachbereich Geschichts- und Kulturwissenschaften, die aus den auf ganz Berlin-Dahlem verteilten Villen ausgezogen sind. Der Gebäudeteil, der sich baulich an die „Silberlaube“3 und die sanierte Bereichsbibliothek der Erziehungswissenschaften anschließt, enthält die neue Campusbibliothek mit einer Nutzfläche von 5580 Quadratmetern, 950 Lese- und Arbeitsplätzen, 12 Gruppenräumen sowie ca. 1 Million Bände, aufgestellt nach RVK. Im sanierten Bau der ehemaligen Bereichsbibliothek Erziehungswissenschaften/Psychologie befinden sich auf sechs Halbetagen die Bestände der RVK-Gruppen A bis D. Im Neubau konzentrieren sich auf den drei Etagen die Bestände der RVKGruppen E bis Z, die Bestände der Ostasiatischen Seminare Japan, China und Korea sowie die Lehrbuchsammlung.

3 Die so genannte „Silberlaube“ wurde 1975-1979 erbaut und dient zusammen mit der „Rostlaube“ als zentraler Hörsaal- und Seminarraum-Komplex der FU.
Das Besondere an der neuen Bibliothek sind zum einen die Heterogenität der vertretenen Fächer und zum anderen die die Herkunft aus den Dahlemer Villen, in denen die Institute und Bibliotheken zuvor residierten. ${ }^{4}$

Für die Institutsangehörigen war der Auszug aus den frei stehenden Institutshäusern mit Balkonen und Gärten, relativer Autonomie und einer Einheit von Professoren, wissenschaftlichen Mitarbeitern, Studierenden, Sekretariat und Bibliothek nicht immer einfach.

\section{Der Umzug}

Charakteristisch für den Fachbereich Geschichts- und Kulturwissenschaften der FU Berlin war die in den vergangenen Jahrzehnten gewachsene Situation, in der die „Kleinen Fächer“ über Dahlem verteilt in Villen angesiedelt waren. Dies hatte auf die logistische Planung große Auswirkungen, weil diese Villen zwar über repräsentative Räumlichkeiten verfügten, aber nie für einen reinen Bibliotheksbetrieb geplant gewesen waren und daher

4 Von den Villen zu den „Lauben“, Mai 2015, noch unveröffentlicht. 


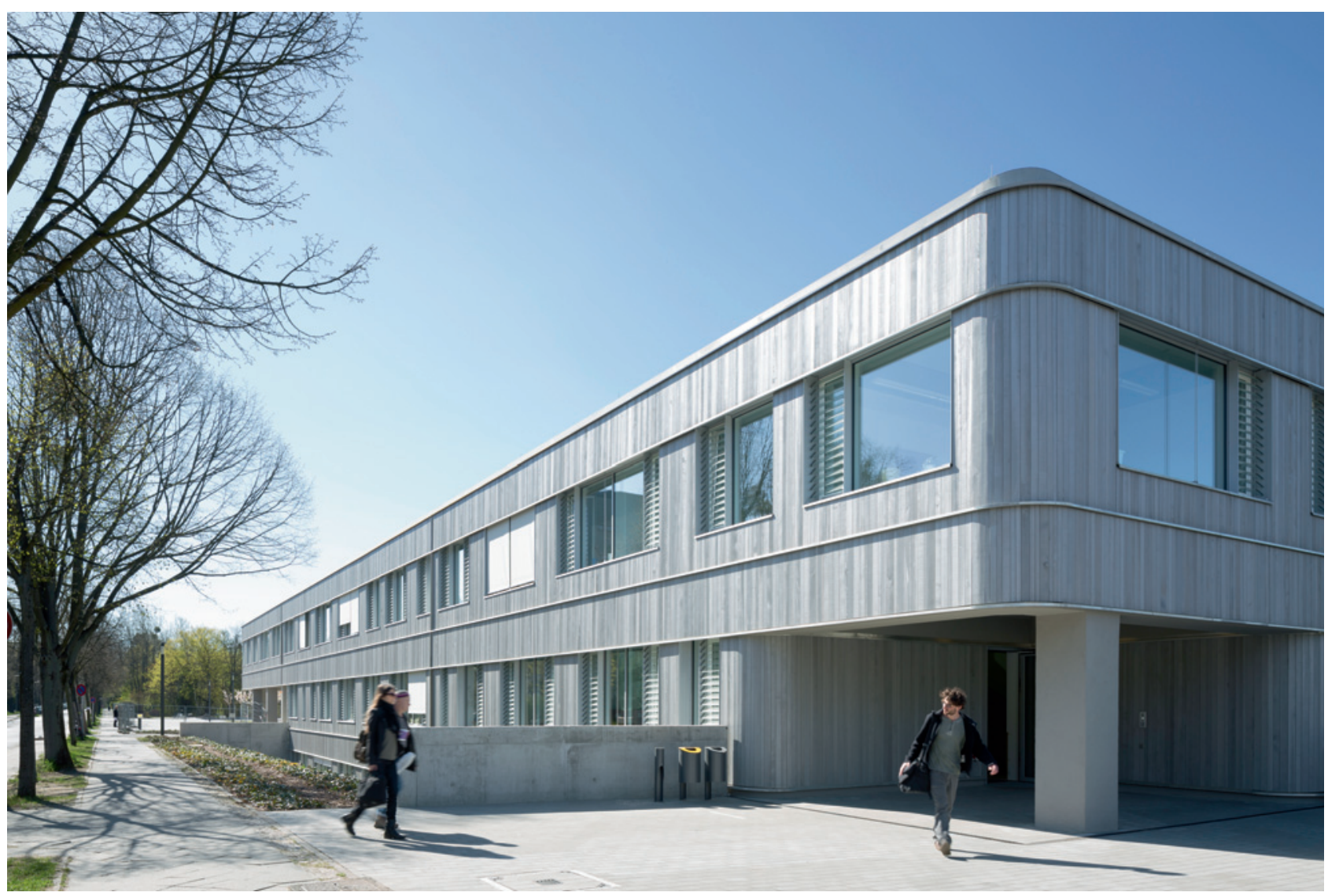

Abb. 2: Ansicht Ost der Campusbibliothek, Architektur: Florian Nagler Architekten (Foto: Stefan Müller-Naumann)

verschiedene, sehr individuelle Lösungen für die Unterbringung der Bestände im Laufe der Jahrzehnte gefunden worden waren. Dies bedeutete etwa, dass sich teilweise über bis zu drei Etagen verteilt Bibliotheksbestände fanden, die alle nach hausspezifischen Systematiken aufgestellt wurden. Im Zuge des Projektes „24 in 1“ wurden diese Bestände zwar alle auf die RVK umgearbeitet, zum Teil konnten sie allerdings aufgrund des fehlenden Platzes vor Ort nicht nach RVK aufgestellt werden. Dies erschwerte für die Speditionen das Einpacken der Bestände nach einer definierten Reihenfolge. Zusätzlich ist ein Großteil der Villen in ruhigen Seitenstraßen gelegen, was die Anfahrt mit Lastkraftwagen durch die Speditionen erschwerte. Vor Ort verfügte keiner der Standorte über Fahrstühle, der Großteil der Gebäude war außerdem nicht barrierefrei zugänglich. Dies machte den Einsatz spezieller Bücherwagen für den Abtransport der Bücher weitgehend unmöglich, weshalb auf Umzugskisten zurückgegriffen werden musste.

Die Vielzahl der Standorte, die innerhalb des sehr knapp bemessenen Umzugszeitraumes von sechs Wochen angefahren werden mussten, und die skizzierten Schwierigkeiten an den einzelnen Quellstandorten führ- ten dazu, dass der Umzug in Kooperation von zwei Speditionsunternehmen durchgeführt wurde. Hierbei fungierte dann eine Firma als Generalunternehmen, das federführend für die Planung verantwortlich zeichnete. Diese Spedition legte fest, dass insgesamt zwei Teams gebildet wurden, die von je einem Projektleiter geführt wurden. An den Quellstandorten wurden die Bestände von festgelegten Teams eingepackt, die von mehreren Fachpackern angeleitet wurden. Auf diese Weise sollte die (vor und während) des Umzugs erworbene Kenntnis des Signatursystems genutzt werden, um ein zügiges Arbeiten beim Einpacken der Bestände sicherzustellen. Am Zielstandort waren entsprechend von beiden Speditionen Fachpacker eingeplant, um die während des Tages angelieferten Meter möglichst schnell in ihre Regale einzuräumen.

In den Villen stellten sich während des Umzugs mitunter Probleme, die Umplanungen vor Ort erforderlich machten. Ein Beispiel war ein Tresor, der im Keller des Instituts für Judaistik wertvolle Bestände beinhaltete. Dieser Tresor entpuppte sich während des Umzuges mit 1,5 Tonnen als dreimal so schwer, wie vorher angenommen. 


\section{Übersicht Belegungsplan von A - Z}

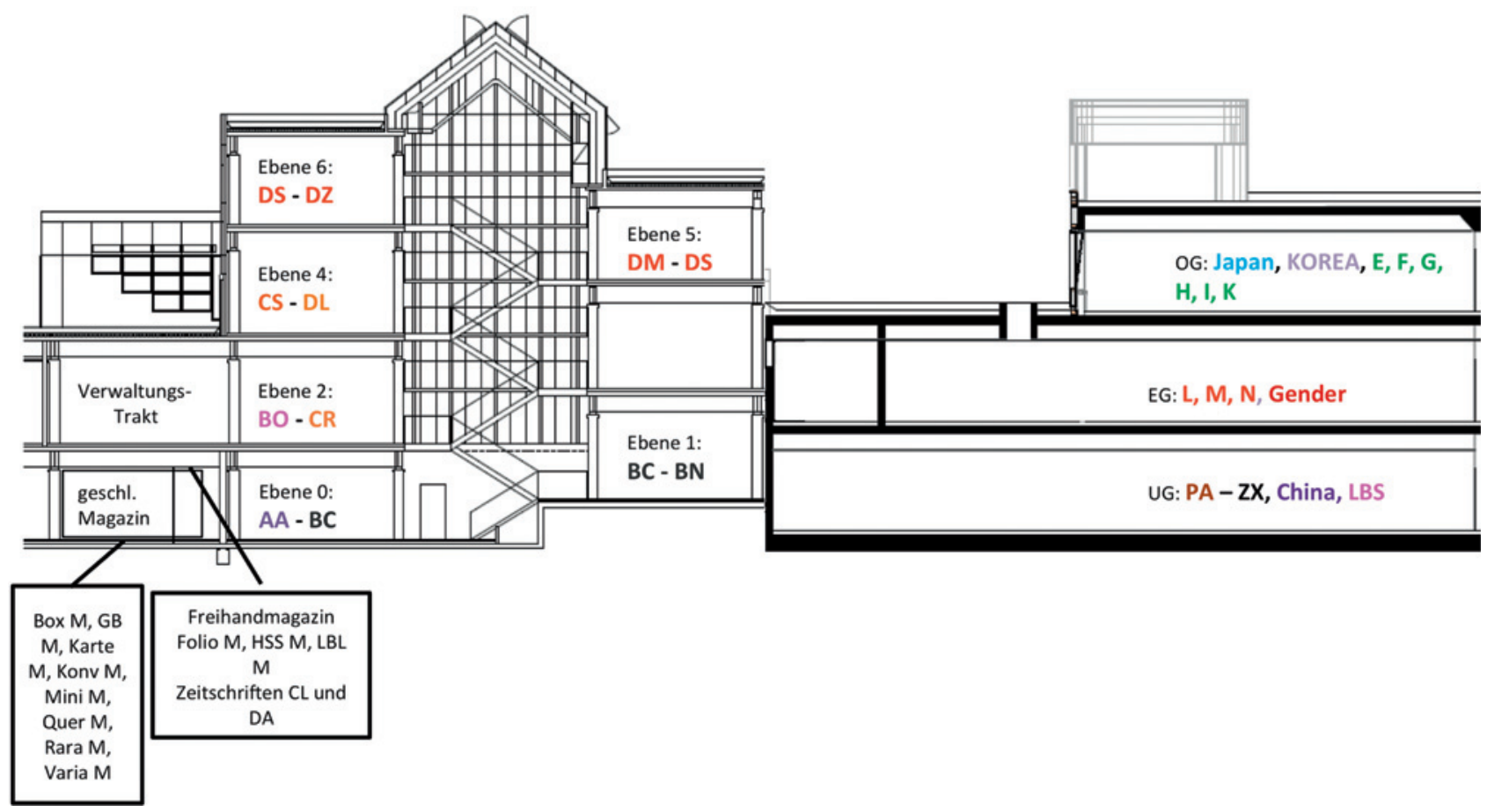

Abb. 3: Übersicht der Belegungsplanung

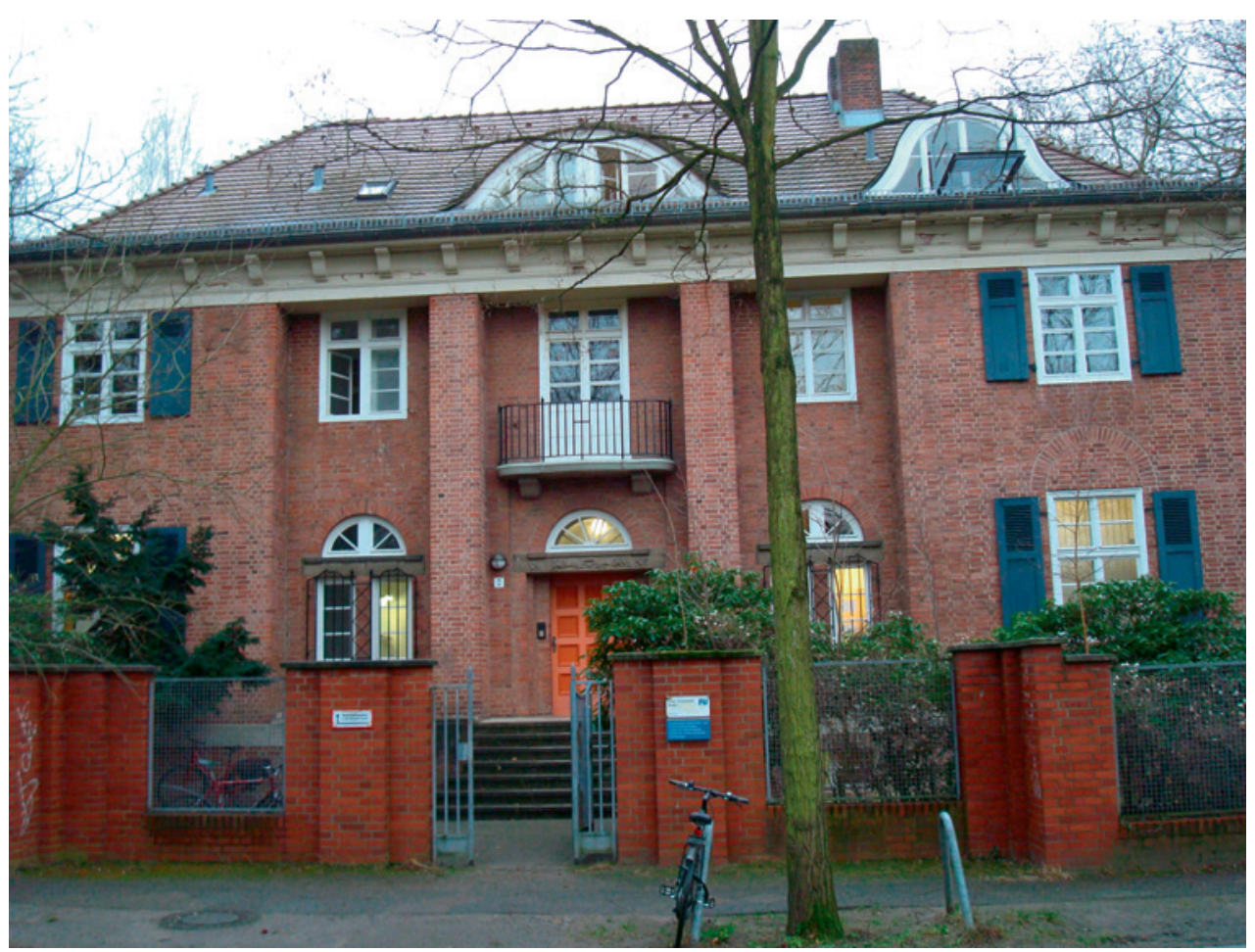

Abb. 4: Beispiel Villa Hüttenweg (Foto: Michael Franke) 


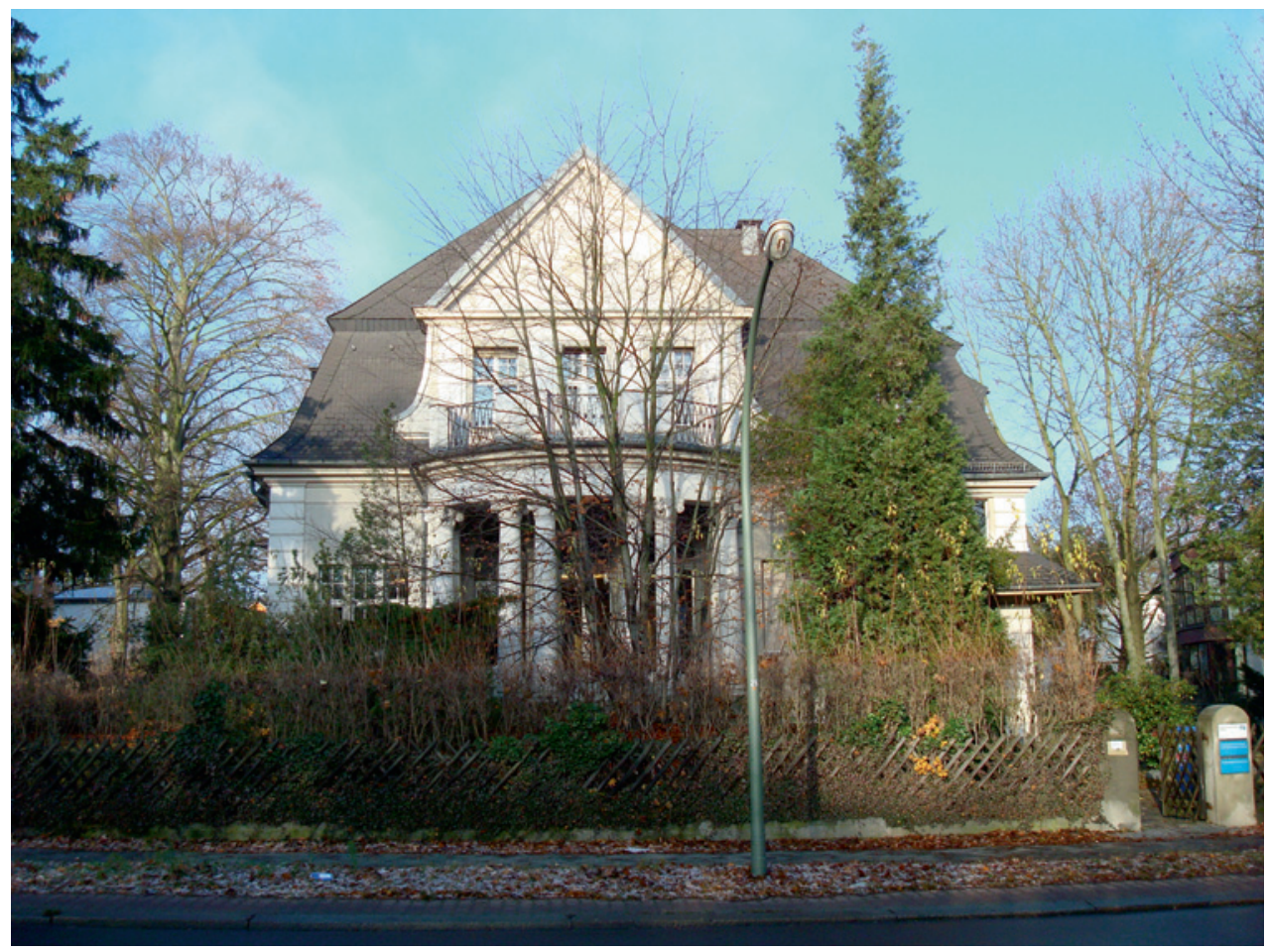

Abb. 5: Beispiel Villa Altensteinstraße 33 (Foto: Michael Franke)

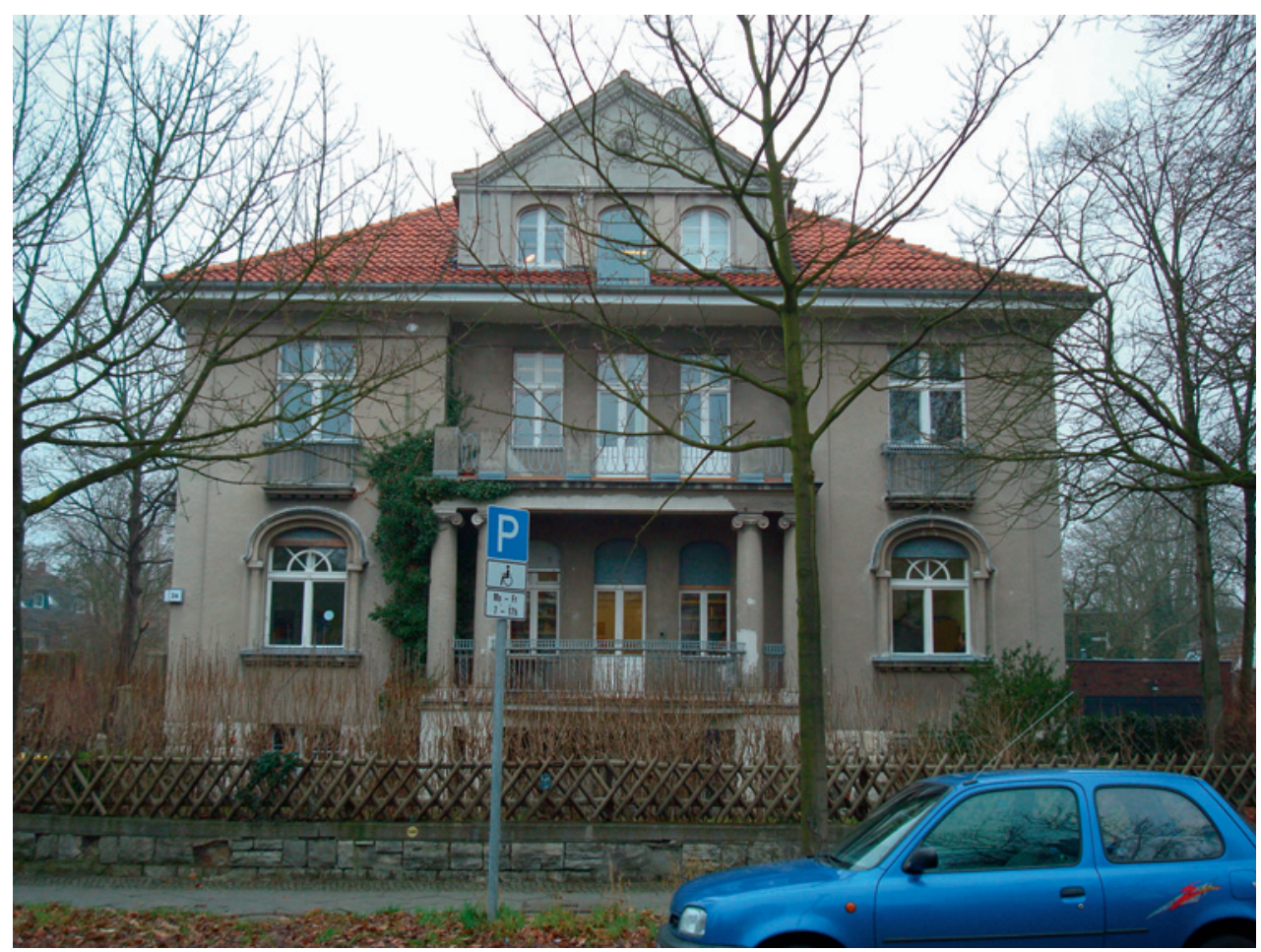

Abb. 6: Bespiel Villa Altensteinstraße 34 (Foto: Michael Franke) 
Herausforderung: Ein

Signatursystem A-Z

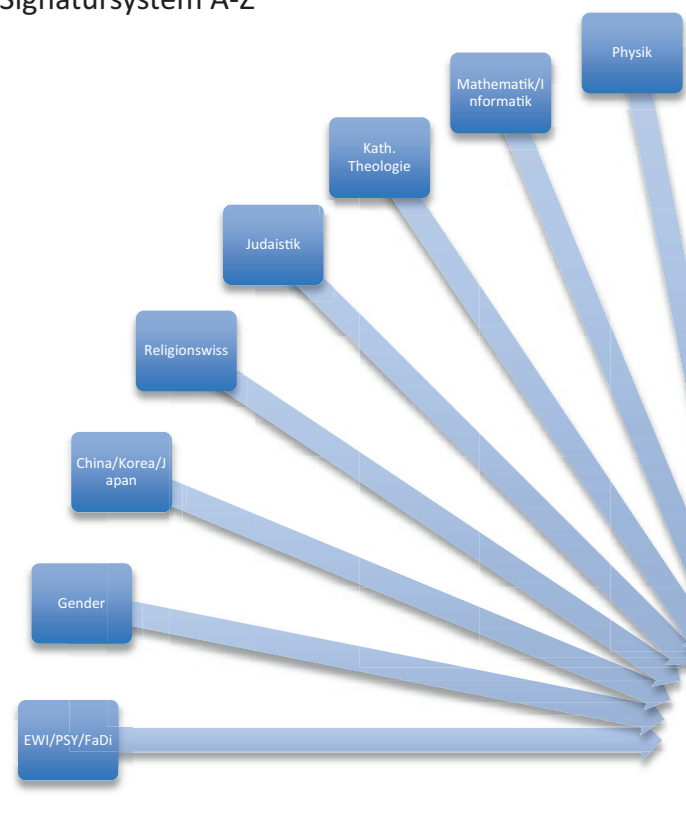

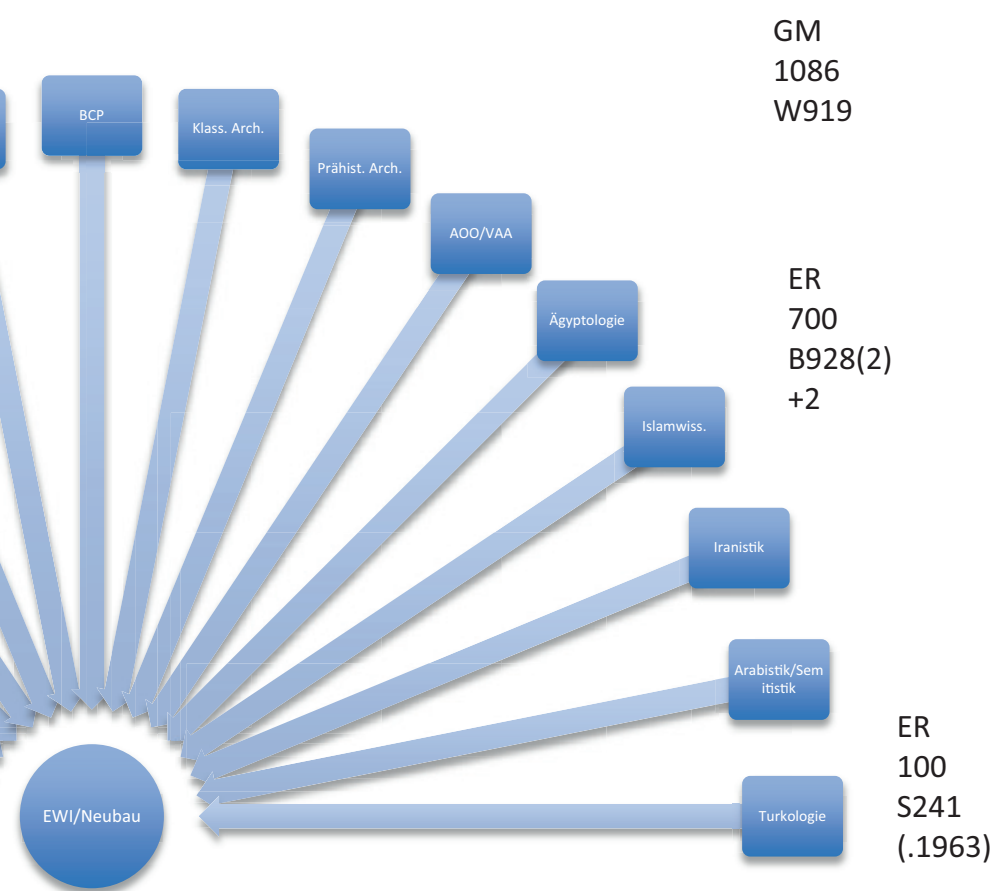

Abb. 7: Illustration des Problems ,Ineinandersortieren"

\subsection{Umzugsplanung und Tests}

Zum Zeitpunkt des Umzugs im März 2015 waren ca. 650000 Medien nach RVK umsigniert, was ein interessantes logistisches Problem darstellte: das Ineinandersortieren der Bestände am Zielort.

Normalerweise sind für eine Regalplanung Excel-Tabellen ausreichend. ${ }^{5}$ Für den komplexen Umzug an der FU Berlin 2015 wurde mit der Firma arTec GmbH Berlin ein visuelles Bestandsplanungssystem namens V:Store entwickelt, welches über eine Schnittstelle mit unserem Bibliothekssystem Aleph verbunden ist. Eine der Schlüsselfunktionen ist die Zusammenführung der Bestände aus unterschiedlichen Standorten mit positiven oder negativen, globalen oder sigelspezifischen Filtermechanismen und die anschließende Darstellung auf der definierten Regalebene. ${ }^{6}$

In der Planungsphase wurde jedoch sehr deutlich, dass für den Gesamtumzug weitaus mehr Informationen in V:Store eingepflegt werden mussten. Dies betraf insbesondere die Breite der einzelnen Medien in den Signaturengruppen. Daher wurden diverse Messteams losgeschickt, die in den einzelnen Bibliotheken genauere

5 Siehe z. B. Smith, Debbi A.: Creating Stack Floor Plans and Signage. In: Technical Services Quarterly 24 (2007), S. 29-39.

6 Näheres hier: Franke, Michael: Spielerisch Bücher platzieren. In: b.i.t. online 16 (2013), S. 118-123.
Messungen durchführten, um das Rücken großer Bestände möglichst zu vermeiden. In der Nachbetrachtung wären eine, maximal zwei durchgängig zuständige Personen für die Messung vorteilhaft gewesen. Außerdem sollte jedes Regal - egal, wie voll es gestellt ist - als ein Regalmeter zählen, um Puffer zu haben.

Um eine Abschätzung zu erhalten, wie viel Zeit für das Einpacken, Transportieren, Auspacken und Integrieren von definierten Beständen einzuplanen ist, wurden im Vorfeld mehrere kleinere Testumzüge durchgeführt. Außerdem dienten die Tests dazu, die Schwierigkeiten eines integrativen Umzugs und die Qualität der Integration durch eine Firma zu ermitteln.

Für die Testumzüge wurden Bestände aus den Bibliotheken der Physik und Chemie im Spätsommer 2014 ausgewählt und die Bestände der Hauptgruppen aus den jeweiligen Bibliotheken konzentriert (RVK-Gruppe U in der Physik; RVK-Gruppen V, W, X, Y in der Chemie). Es wurden aus der Physik 50 laufende Meter verschoben, aus der Chemie 80 laufende Meter. Die Vorbereitung eines Regalhandbuches, anhand dessen man die Bestände an die richtige Stelle im Regal einstellen konnte, war unabdingbar für ein zügiges Einräumen der Bestände. Im Regalhandbuch wurde die Startsignatur des vorliegenden Regalbodens aufgeführt, so dass die Packer schnell erkennen konnten, welche Bücher auf diesem Boden untergebracht werden mussten. Die zeitlichen Probleme bei den Testumzügen lagen in der Vorbereitung des Regal- 


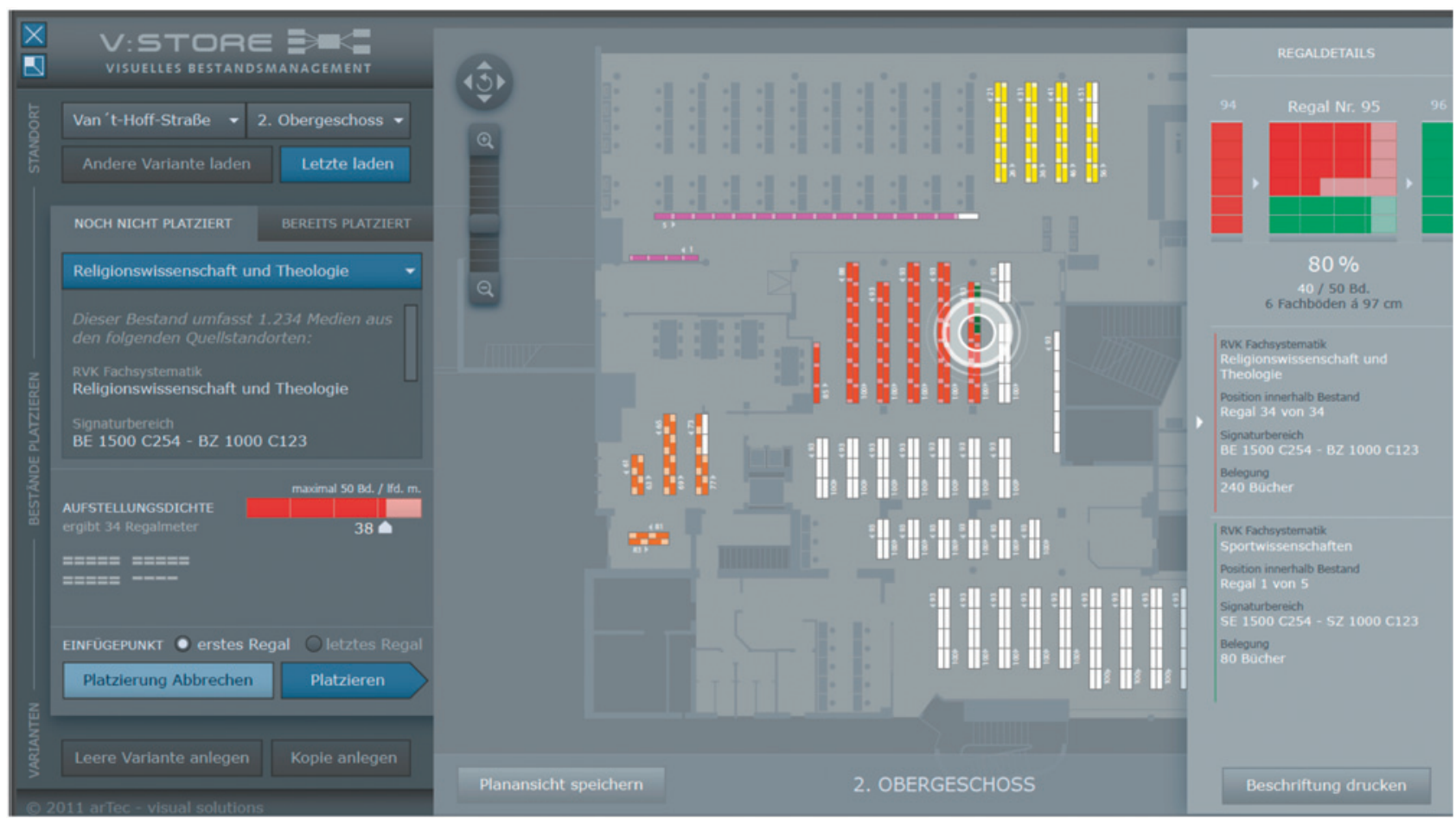

Abb. 8: V:Store

handbuches, welches per Hand durch Ausmessen, Abschätzen und Festlegen einer potentiell passenden Startsignatur pro Boden ermittelt werden musste.

Mit Abschluss der Tests in den Naturwissenschaften stand fest, dass ein Packer im Schnitt ca. vier Meter Bü- cher pro Stunde korrekt in größere bereits aufgestellte Bestände einsortieren kann. Der Anteil der integrativen Bestände in der neuen Campusbibliothek lag bei ca. 40 Prozent. Dies bedeutete, dass etwa 60 Prozent der Bestände als Hauptbestände ohne Integration in die leeren

\section{MINT}

\section{August / September 2014}

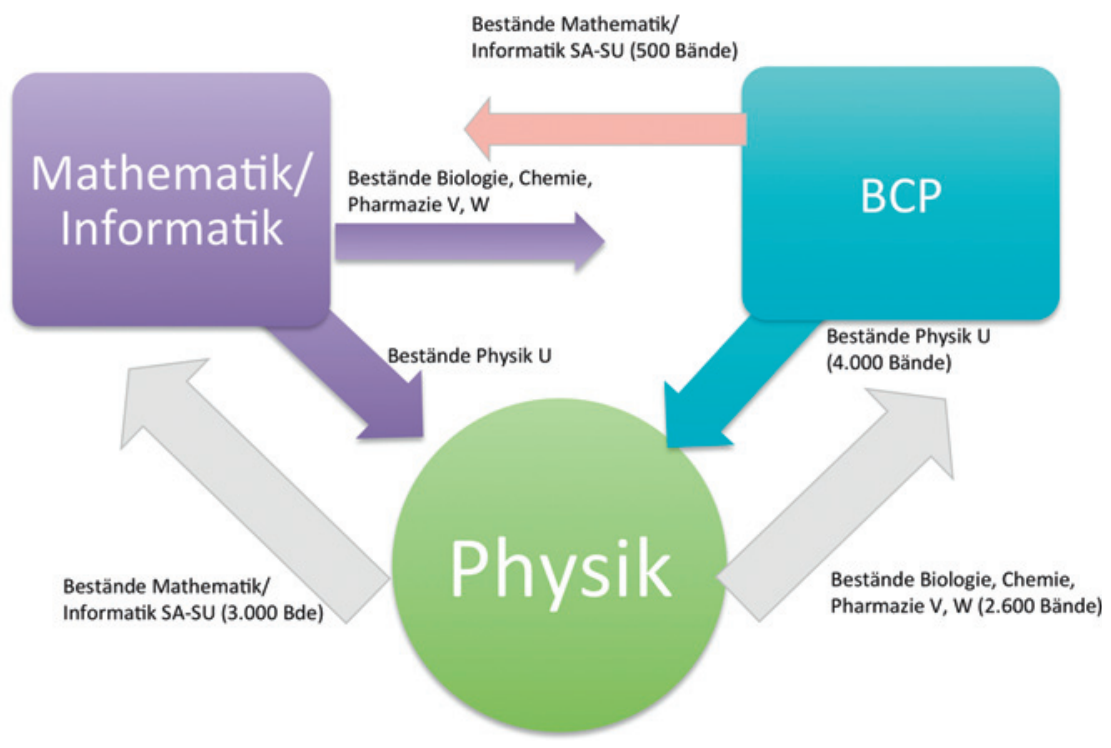

Abb. 9: Vorabintegration MINT 
Regale eingeräumt und kleinere Anteile im Umfang von insgesamt etwa 40 Prozent aus mehreren Standorten im Anschluss integriert werden konnten.

Davon ausgehend wurde beschlossen, möglichst viele kleinere Nebenbestände aus den verschiedenen Standorten in den Bibliotheken zusammenzuführen, welche die entsprechenden RVK-Gruppen als Hauptbestände enthielten. Die Idee dabei war, den integrativen Teil des Gesamtumzugs so gering wie möglich zu halten, um Zeit beim Einsortieren der Bestände zu sparen. Da die Sanierung des Altbaus bereits Anfang 2015 abgeschlossen war, konnte dieser Teil für die Vorabintegrationen genutzt werden. Aufgrund des Trennens der Bestände musste im großen Umzug nur noch der Neubau der Campusbibliothek beliefert werden. Das war logistisch eine relevante Erleichterung, da die Lieferzonen vor und das Bewegen der Bestände innerhalb der Bibliothek damit deutlich beschränkt werden konnten.

\subsection{Vorabintegrationen}

Die Vorabintegrationen wurde in zwei Schritten vollzogen. Als erstes wurden im November 2014 im Außenmagazin Mecklenburgische Straße periphere Bestände der Villenbibliotheken zusammengezogen. Die Aufteilung in Kernbestand und Peripherie wurde in Abstimmung mit den Wissenschaftlern anhand von Excel-Tabellen bestimmt, welche die Verteilung der RVK im jeweiligen Haus zeigte. Dies hatte den vorteilhaften Nebeneffekt, dass in den Villen Platz für die korrekte RVK-Aufstellung gewonnen wurde.

Im zweiten Schritt wurden im Januar und Februar 2015 die RVK-Gruppen A-D in die sanierte ehemalige Be- reichsbibliothek Erziehungswissenschaft und Psychologie integriert. Im Altbau der Campusbibliothek wurden von Januar bis Februar 2015185000 Medien in den Regalen aufgestellt. Neben der normalen Herausforderung, über einen Fahrstuhl die Bestände auf insgesamt sechs Ebenen zu verteilen, kam hinzu, dass der Einzug der Medien auf eine Baustelle erfolgte. Die Räumlichkeiten der Bibliothek waren noch nicht komplett fertig gestellt, was einen erhöhten Absprachebedarf mit dem Architekten, der Technischen Abteilung der FU und natürlich auch mit den Handwerkern vor Ort erforderlich machte. So konnten manchmal bewährte Lieferwege nicht genutzt werden, da der Teppich verlegt werden musste, oder Kartons und Bücherwagen mussten verschoben werden, um das Verlegen von Kabeln zu ermöglichen. Auch das Reinigen der Regale in regelmäßigen Abständen musste abgeklärt werden. Außerdem mussten teilweise vor dem Bücherumzug die entsprechenden Regale noch aufgebaut werden, in denen vorher die Bücher an anderen Standorten untergebracht waren.

Der größte Teil für diese Vorabintegration kam aus dem Außenmagazin in der Mecklenburgischen Straße. Dieser Standort sollte bis zum Beginn des Umzugs im März 2015 geräumt werden. Gleichzeitig waren im Interimsstandort der Bibliothek für Erziehungswissenschaft in der Van'tHoff-Straße 6 größere Bestände der Signaturen A-D in Benutzung. Zusätzlich wurde die Räumung der Bibliotheken für Judaistik und Katholische Theologie in den Altbau in den letzten beiden Wochen vor dem Umzug terminiert, weil diese zum Großteil aus Büchern der Signaturguppe B der RVK bestanden und mit ihrer Integration in den Bestand des Altbaus zwei Villenstandorte vor dem großen Umzug komplett eingearbeitet werden konnten.

Tabelle 1: Übersicht der Vorabintegration RVK-Monographien

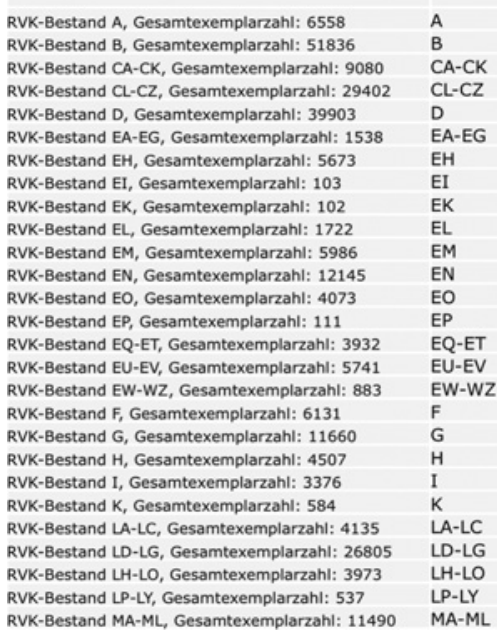

\begin{tabular}{|c|c|c|c|c|c|c|c|c|c|c|c|c|c|c|c|c|c|}
\hline 8028 & 812 & 814 & 8228 & 867 & 871 & 877 & 8798 & 8858 & $896 \varepsilon$ & $898 \mathrm{~s}$ & 9129 & $930 \mathrm{~B}$ & $B C P \quad E$ & EWI Plus $\mathrm{N}$ & Meck24a & Silberlaube & $\begin{array}{l}\text { Summ } \\
\text { e }\end{array}$ \\
\hline 115 & $5 \quad 558$ & 3238 & $8 \quad 116$ & 62 & 21 & $1 \quad 396$ & $96 \quad 491$ & 23 & 39 & 87 & 321 & 114 & 318 & 1213 & $3 \quad 2523$ & $3 \quad 3$ & $3655 \varepsilon$ \\
\hline 1265 & 57092 & 22 & 579 & 94343 & & 11710 & 1046251 & 11903 & 455 & 7 & 177 & 5175 & 7 & 4195 & 10152 & 128 & $5183 \epsilon$ \\
\hline 30 & $\begin{array}{l}0 \quad 757 \\
0\end{array}$ & 83 & 10 & $\begin{array}{ll}0 & 122\end{array}$ & 2 & $1 \quad 209$ & D9 345 & 217 & 5 & 295 & 18 & 676 & 87 & 1706 & 4514 & 5 & $908 \mathrm{C}$ \\
\hline 5 & 31 & 18 & 3 & 3 & 0 & 0 & 32 & 3 & 0 & 21 & 2 & 121 & 80 & 8255 & 20823 & 0 & 29402 \\
\hline 2 & 98 & 8 & 4 & 4 & 0 & 19 & 22 & 0 & 0 & 20 & 11 & 45 & 36 & 14745 & 24893 & 0 & 39903 \\
\hline 54 & 49 & 0 & 4 & 4 & 5 & 56 & 121 & 7 & 14 & 2 & 49 & 13 & 4 & 322 & 838 & 0 & 1538 \\
\hline 16 & $5 \quad 1534$ & 0 & 2 & 2 & 0 & 150 & 29 & 1 & 0 & 0 & 3778 & 1 & 0 & 12 & 149 & 1 & 5673 \\
\hline 0 & 2 & 0 & 0 & 0 & 0 & 0 & 7 & 0 & 1 & 0 & 70 & 0 & 0 & 9 & 7 & 0 & 103 \\
\hline 8 & 2 & 0 & 0 & 0 & 0 & 1) & 4 & 1 & 0 & 0 & 51 & 3 & 0 & 7 & 9 & 0 & 102 \\
\hline 353 & 482 & 0 & 2 & 2 & 1 & 196 & 512 & 13 & 55 & 0 & 52 & 8 & 0 & 2 & 46 & 0 & 1722 \\
\hline 2249 & 68 & 0 & 0 & 0 & 5 & 169 & 92218 & 573 & 87 & 4 & 3 & 30 & 0 & 4 & 576 & 0 & 5986 \\
\hline 90 & 4938 & 0 & 1 & 1 & 1 & 254 & $4 \quad 6730$ & 11 & 28 & 0 & 37 & 2 & 0 & 2 & 51 & 0 & 12145 \\
\hline 54 & $\begin{array}{l}4 \quad 37 \\
\end{array}$ & 0 & 16 & & 3 & 0 & $9 \quad 261$ & 13 & 3581 & 0 & 0 & 4 & 0 & 1 & 94 & 0 & 4073 \\
\hline 5 & 10 & 0 & 0 & 0 & 0 & 0 & 41 & 0 & 31 & 0 & 0 & 0 & 0 & 0 & 24 & 0 & 111 \\
\hline 209 & 21 & 2 & 2 & 2 & 2 & 126 & 112 & 1 & 56 & 21 & 57 & 0 & 4 & 1184 & 2134 & 0 & 3932 \\
\hline 188 & 381 & 0 & 1 & 0 & 0 & $\begin{array}{ll}0 & 4721\end{array}$ & 200 & 0 & 5 & 0 & 120 & 1 & 0 & 3 & 121 & 0 & 5741 \\
\hline 595 & 1 & 0 & 3 & 0 & 0 & 077 & 25 & 0 & 9 & 0 & 8 & 0 & 0 & 2 & 163 & 0 & 883 \\
\hline 177 & 62 & 3 & 651 & 29 & 9 & 120 & 189 & 32 & 247 & 5 & 13 & 53 & 29 & 542 & 3972 & 7 & 6131 \\
\hline 5 & 88 & 2 & 5 & 2 & 2 & 65 & 110 & 111 & 4 & 5 & 57 & 463 & 8 & 2780 & 7952 & 3 & $1166 \mathrm{C}$ \\
\hline 7 & 69 & 22 & 0 & 0 & 0 & 49 & 49 & 34 & 0 & 6 & 13 & 24 & 54 & 1261 & 2919 & 0 & 4507 \\
\hline 0 & 355 & 11 & 4 & 2 & 2 & 57 & 176 & 31 & 1 & 3 & 13 & 49 & 23 & 560 & 2091 & 0 & $337 \epsilon$ \\
\hline 2 & 1 & 2 & 0 & 0 & 0 & 35 & 14 & 2 & 2 & 1 & 73 & 22 & 15 & 14 & 400 & 0 & 584 \\
\hline 477 & 736 & 0 & $\begin{array}{l}0 \\
0\end{array}$ & & 1315 & 359 & 242 & 15 & 22 & 1 & 257 & 13 & 16 & 457 & 1208 & 0 & 4135 \\
\hline 626 & 38 & & 016949 & & 4205 & 68 & 58 & 4 & 5169 & 1 & 12 & 11 & 7 & 99 & 1707 & 1 & 2680 ! \\
\hline 180 & 376 & 3 & $\begin{array}{l}3 \quad 438\end{array}$ & & $\begin{array}{ll}5 & 16\end{array}$ & 257 & 205 & 3 & 60 & 5 & 91 & 62 & 5 & 309 & 1811 & 2 & 3973 \\
\hline 4 & 42 & 2 & 4 & 0 & 0 & 22 & 76 & 27 & 6 & 1 & 5 & 22 & 0 & 98 & 227 & 0 & 537 \\
\hline 19 & 4598 & 17 & 1 & & 4 & 892 & 395 & 350 & 1 & 2 & 315 & 374 & 3 & 863 & 3655 & & $1149 c$ \\
\hline
\end{tabular}




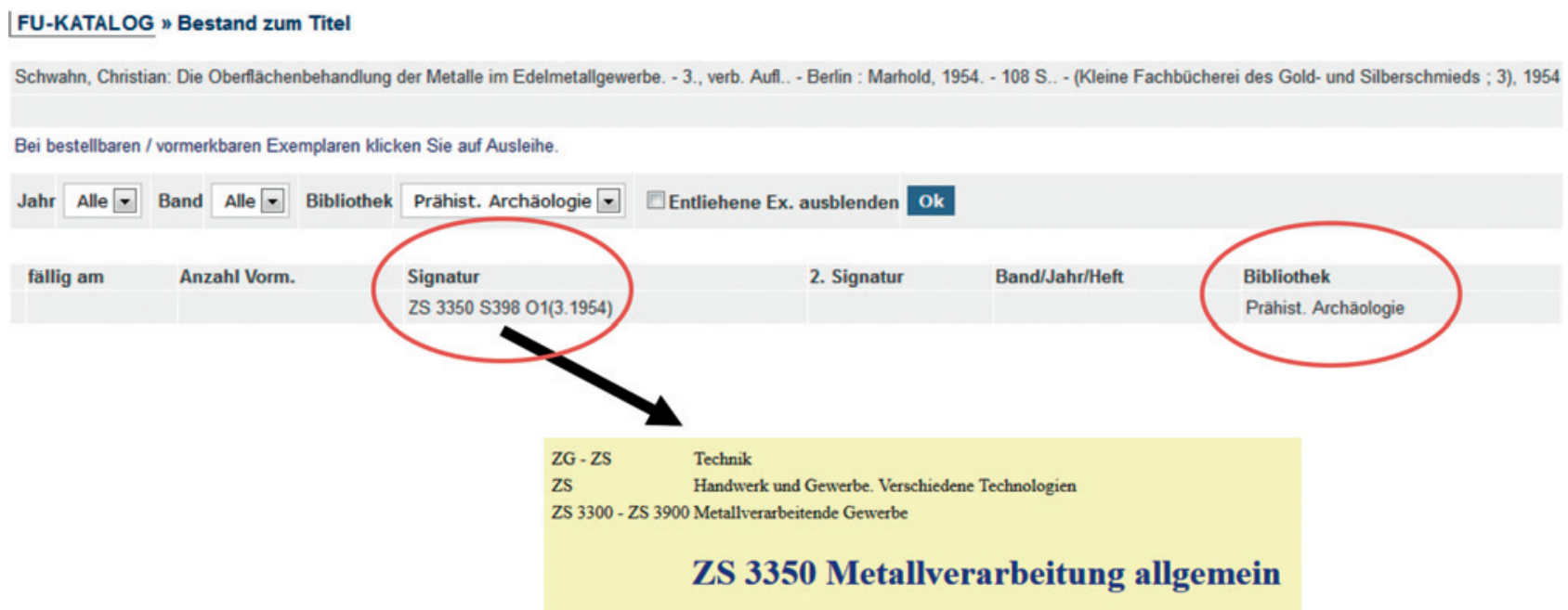

Abb. 10: Beispiel für peripheren Bestand

Diese Vorabumzüge mussten genau aufeinander abgestimmt werden, da auch noch nicht nach RVK umsignierter Altbestand von der Mecklenburgischen Straße in die Van't-Hoff-Straße 6 transportiert werden sollte. Dies setzte aber den vorherigen Umzug der Bestände A-D von der Van't-Hoff-Straße 6 in den Altbau der Campusbibliothek voraus und musste wiederum aufgrund der vorhandenen Personalkapazität der beteiligten Spedition vor der Räumung von Judaistik und Katholischer Theologie erfolgen.

Bei diesem Umzug der Bestände A-D wurden die integrativen Einstellarbeiten bis zum Beginn des großen Umzugs komplett von der Firma übernommen, die auch das Umziehen der Bestände aus den jeweiligen Standorten übernommen hatte. In diesem Zusammenhang wurde deutlich, dass das Integrieren der Medien mehr Zeit in Anspruch nehmen würde, als es der Zeitplan für den großen Umzug vorsah. Ein weiteres unterschätztes Problem waren ausfallende Aufzüge, welches durch die Bereitstellung von Fahrstuhlwächtern zur sachgerechten Bedienung sowie eines Bereitschaftsdienstes von Wartungstechnikern gelöst werden konnte.

Im Monat vor dem eigentlichen, großen Umzug wurde zusätzlich eine weitere Spedition mit dem Abtransport der Signaturgruppen A-D aus den übrigen Villenstandorten in den Altbau betraut, wobei hierbei in zwei Schritten vorgegangen werden musste. Es wurden wie bei der ersten Vorabintegration Mengen definiert, die pro Signaturgruppe innerhalb der RVK als Kernbestand der jeweiligen Bibliothek betrachtet wurden, und Gruppen, deren Zahl so gering ausfiel, dass diese als peripherer Bestand gelten konnten. Dieser periphere Bestand wurde zuerst aus den Villenbibliotheken entnommen und im Altbau integriert, während die definierten Kernbestände erst in der Woche vor dem großen Umzug aus den Bibliotheken entnommen wurden, um für die Benutzung möglichst lange zur Verfügung zu stehen.

Die Vorabintegration kleinerer Bestände in den $\mathrm{Na}$ turwissenschaften und das Vorziehen des Umzugs der Signaturengruppen A-D in den Altbau der Campusbibliothek waren wichtige Schritte in der Vorbereitung des großen Umzugs in den Neubau.

\subsection{Organisation}

Mit Beginn der Vorabintegration der naturwissenschaftlichen Bestände wurde die Notwendigkeit einer engen Abstimmung des weiteren Umzugsgeschehens mit der Technischen Abteilung der FU Berlin deutlich. Diesem Umstand wurde Rechnung getragen, indem eine regelmäßig tagende Arbeitsgruppe mit Vertretern der Technischen Abteilung, der Leitung der UB der FU Berlin, der Bibliotheksleitung und den Umzugskoordinatoren etabliert wurde. Diese Runde tagte ab diesem Zeitpunkt in zweiwöchentlichem Turnus. Inhalt dieser Gruppe war die kontinuierliche Besprechung der Fortschritte des Umzugsgeschehens und eine ständig aktualisierte Planung des restlichen Umzugsgeschehens, die auf die Entwicklungen an den einzelnen Standorten flexibel reagieren konnte. Auf diese Weise wurde es ermöglicht, auf schneller als geplant erfolgte Teilumzüge mit dem Vorziehen anderer Teilschritte zu reagieren. Dies trug der dynamischen Situation innerhalb des Projektes Rechnung, in dem Bestände noch während der laufenden Planung bearbeitet wurden.

In der „heißen Phase“ des großen Umzugs wurde im operativen Alltag die Verantwortung für die Standorte 


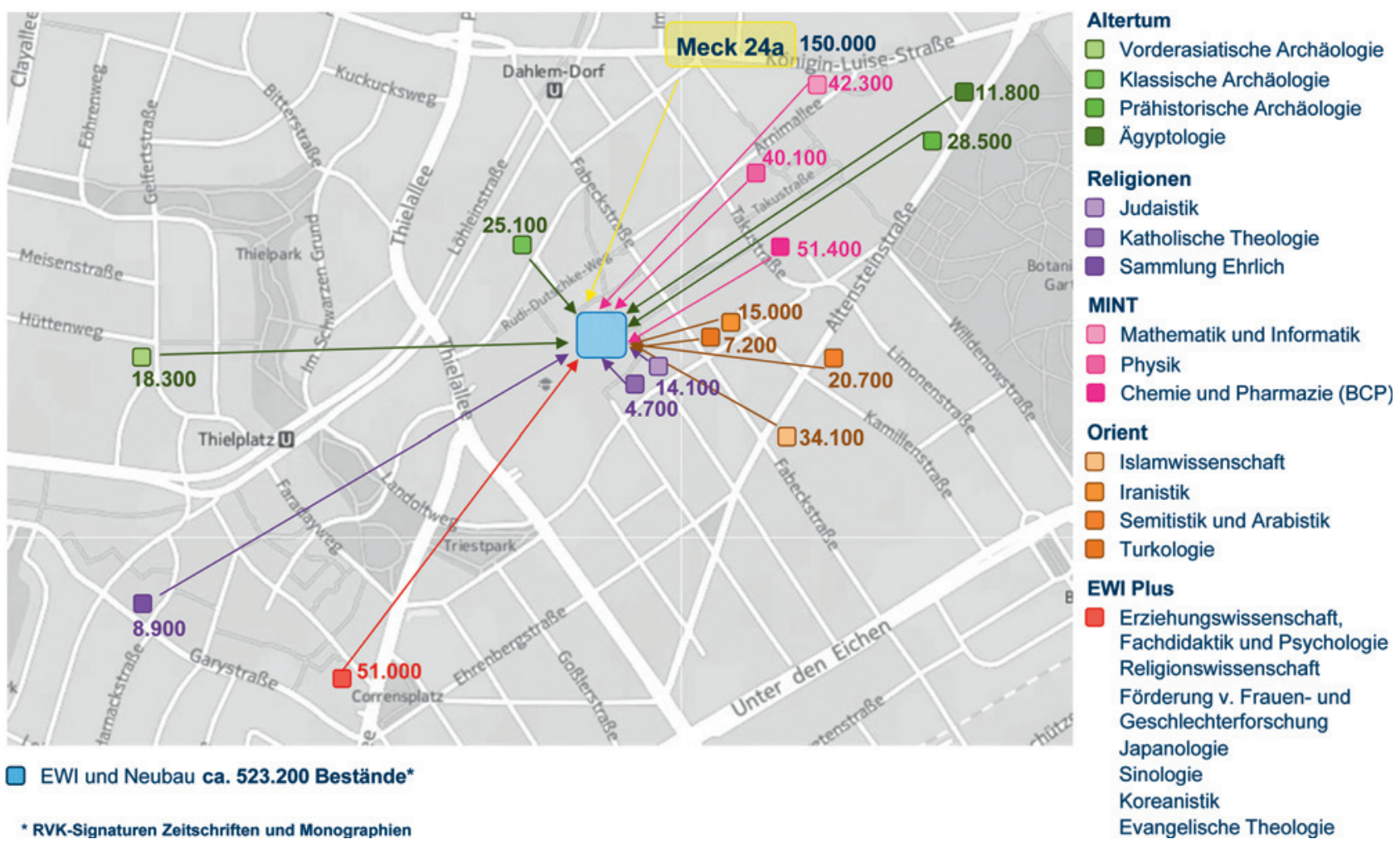

Abb. 11: Herkunft der Bestände (Quelle: Maria Jahnke)

auf drei Personen aufgeteilt: (Bibliotheksleitung: Altbau, Umzugskoordinatorin: Neubau, Umzugskoordinator: Quellstandorte). Ferner wurde eine Früh- und Spätschicht vereinbart, da die Speditionen um 7.30 Uhr begannen und die Einstelldienste bis 19.30 Uhr andauerten. So wurde sichergestellt, dass während des gesamten Umzugsbetriebs immer mindestens eine Person mit Entscheidungsbefugnissen vor Ort war. Diese drei Personen zeichneten auch verantwortlich für die Organisation der Sonderdienstpläne für Einstelldienste, Revision, Fahrstuhl- und Türwächter, Pausenraum, Auskunft und Wegweiser. Für die Regalplanung mit V:Store und die Rückfragen standen zwei gesonderte Experten zur Verfügung. Im Neubau wurde eine Umzugszentrale eingerichtet, die immer mit mindestens einer Ebenenkoordination besetzt war, um Fragen zur Systematik, Reihenfolge und anderen Problemen zu beantworten. Diese Schlüsselpersonen wurden mit Diensthandys zur ständigen Erreichbarkeit ausgestattet.

Auf Seiten der Technischen Abteilung waren zwei Personen für die Auftragsvergabe, die Auftragsdurchführung und den Kontakt zu den beiden Speditionen verantwortlich. Von Seiten der Spedition gab es einen Projektleiter, der die konkrete Detailplanung der einzelnen Umzugsportionen und die Markierung der Bestände an den Quellstandorten vornahm. Zusammen mit der Umzugskoordination trafen sich diese Personen jeden Freitag zu einer Rückschau der Woche. Von jeder Spedition war jeweils ein Kolonnenführer anwesend, der als ständiger Ansprechpartner vor Ort war und der kurzfristige Planänderungen und Justierungen ermöglichte. Jeden Tag wurde mit den Kolonnenführern eine Besprechung zum Stand der Dinge durchgeführt.

Gleichzeitig wurden klare Kommunikationswege für alle Beteiligten festgelegt. So sollten sich alle bibliothekarischen Umzugshelfer mit Fragen an die Ebenenkoordination wenden. Diese hatte als Ansprechpersonen die Umzugskoordinatoren. Nur die Koordinatoren hielten den Kontakt zur Spedition.

Die genaue Terminplanung für die Umzüge der einzelnen Teilbibliotheken in die Campusbibliothek wurde in enger Absprache zwischen der Technischen Abteilung und den einzelnen Fachbibliotheken durchgeführt. Hierbei musste sowohl auf die durch logistische Notwendigkeiten vorgegebenen festen Umzugstermine, die vor die allgemeine Schließzeit der Bibliotheken fielen, Rücksicht genommen werden als auch an Standorten, für die solche Vorgaben nicht bestanden, mit dem wissenschaftlichen Personal gesonderte Absprachen getroffen werden, wenn die entsprechenden Bestände vorab aus den Biblio- 
Tabelle 2: Ablaufplanung (Quelle: Peter Saal)

\begin{tabular}{|c|c|c|c|c|c|c|c|}
\hline Nr. & & Vorgangsname & Ifm Bücher & Ausgangsort & Anfang & Ende & Dauer \\
\hline 1 & & Logistik Step 1 - Räumung Meck24a & & & Mon 20.10.14 & Fre 27.02.15 & 85 Tage? \\
\hline 2 & $\checkmark$ & Step 1.1 Ebene 2 / 4 & $1.321 \mathrm{~m}$ & & Mon 20.10.14 & Die 18.11.14 & 22 Tage \\
\hline 3 & $\checkmark$ & Regalaufbau 2.304 m & & & Mon 20.10.14 & Mit 29.10.14 & 8 Tage \\
\hline 4 & $\checkmark$ & Bezug Signatur CT - DL (Ebene 4) & $751 \mathrm{~m}$ & & Mon 10.11 .14 & Die 18.11.14 & 7 Tage \\
\hline 5 & $\checkmark$ & Bezug Signatur CA - CN (Ebene 2) & $570 \mathrm{~m}$ & & Mon 10.11.14 & Die 18.11.14 & 7 Tage \\
\hline 6 & $\checkmark$ & Räumung der Regale Keller innerhalb der Meck 24a & & & Mon 17.11.14 & Fre 28.11 .14 & 10 Tage \\
\hline 7 & $\checkmark$ & Step 1.2 Ebene 5 & $133 \mathrm{~m}$ & & Mit 19.11.14 & Mit 26.11.14 & 6 Tage \\
\hline 8 & $\checkmark$ & Regalaufbau $288 \mathrm{~m}$ & & & Mit 19.11.14 & Don 20.11.14 & 2 Tage \\
\hline 9 & $\checkmark$ & Bezug Signatur DM - DP (Ebene 5) & $133 \mathrm{~m}$ & & Mon 24.11.14 & Mit 26.11.14 & 3 Tage \\
\hline 10 & $\checkmark$ & Step 1.3 Ebene 6 & $84 \mathrm{~m}$ & & Fre 21.11.14 & Mit 26.11.14 & 4 Tage \\
\hline 11 & $\checkmark$ & Regalaufbau 288 m & & & Fre 21.11.14 & Fre 21.11.14 & $1 \mathrm{Tag}$ \\
\hline 12 & $\checkmark$ & Bezug Signatur DQ - DZ (Ebene 6) & $84 \mathrm{~m}$ & & Mon 24.11.14 & Mit 26.11.14 & 3 Tage \\
\hline 13 & $\checkmark$ & Vorlaufzeit Ausschreibung Step 1.4 und Step 1.5 & & & Die 11.11.14 & Mon 08.12.14 & 20 Tage \\
\hline 14 & $\checkmark$ & Step 1.4 Bezug Boltzmannstraße 20 - Altsignaturen & $900 \mathrm{~m}$ & & Die 09.12.14 & Don 18.12.14 & 8 Tage \\
\hline 15 & $\checkmark$ & Step 1.5 Bezug Silberlaube Außenmagazin EL - Z & $1.500 \mathrm{~m}$ & & Die 09.12.14 & Don 18.12.14 & 8 Tage \\
\hline 17 & $\checkmark$ & Angebotseinholung Regalaufbau und Umzug & & & Mit 19.11.14 & Mit 17.12.14 & 20 Tage \\
\hline 18 & $\checkmark$ & Regalaufbau $2.052 \mathrm{~m}$ & & & Mon 05.01 .15 & Die 13.01.15 & 7 Tage \\
\hline 19 & $\checkmark$ & Bezug Signatur BD - BW (Ebene 1) & $350 \mathrm{~m}$ & & Mit 14.01.15 & Die 20.01.15 & 5 Tage \\
\hline 20 & & Step 1.7 und Step 1.8 & & & Mit 10.12.14 & Fre 30.01.15 & 28 Tage? \\
\hline 21 & 罒 & Vorlaufszeit Ausschreibung Step 1.7 und Step 1.8 & & & Mit 10.12.14 & Mit 21.01.15 & 20 Tage \\
\hline 22 & & Step 1.7 Ebene 0 & $90 \mathrm{~m}$ & & Mit 21.01.15 & Mon 26.01.15 & 4 Tage? \\
\hline 23 & & Regalaufbau 1.080 m & & & Mit 21.01.15 & Fre 23.01 .15 & 3 Tage \\
\hline 24 & & Bezug Signatur A - BC (Ebene 0) & $90 \mathrm{~m}$ & & Mon 26.01.15 & Mon 26.01.15 & $1 \mathrm{Tag}$ \\
\hline 25 & & CL Ebene 2 -> Ebene 0 Magazin & & & Fre 23.01.15 & Fre 23.01 .15 & $1 \mathrm{Tag} ?$ \\
\hline 26 & & Step 1.8 Ebene 2 & & & Mit 21.01.15 & Fre 30.01.15 & 8 Tage \\
\hline 27 & $\checkmark$ & Regalaufbau 720 m & & & Mit 21.01.15 & Fre 30.01 .15 & 8 Tage \\
\hline 28 & & Bücher rücken - Signatur CA - CN & $570 \mathrm{~m}$ & & Mon 26.01.15 & Die 27.01.15 & 2 Tage \\
\hline 29 & & Bezug Signatur (Ebene 2) & $300 \mathrm{~m}$ & & Mit 28.01.15 & Mit 28.01.15 & $1 \mathrm{Tag}$ \\
\hline 30 & & Bücher rücken Ebene 1, 4, 5 und 6 (Integration light) & ca. $968 \mathrm{~m}$ & & Mit 28.01.15 & Fre 30.01 .15 & 3 Tage \\
\hline 31 & & Step 1.9 Finale Räumung & & & Don 08.01.15 & Fre 27.02.15 & 37 Tage \\
\hline 34 & & Räumung sämtliches Mobiliar Meck24a & & & Don 05.02 .15 & Fre 06.02.15 & 2 Tage \\
\hline 35 & & Signatur A-D Van't Hoff 6 -> EWI & $1.600 \mathrm{~m}$ & & Mon 16.02 .15 & Fre 20.02.15 & 5 Tage \\
\hline 36 & & Altsignaturen + AP von Meck24a -> Van't Hoff 6 & $550 \mathrm{~m}$ & & Mon 23.02.15 & Mit 25.02.15 & 3 Tage \\
\hline 37 & & Auszug OPAC-Plätze Van't Hoff 6 (2.0G) -> Möbellager & 27 Plätze & & Fre 27.02 .15 & Fre 27.02 .15 & $1 \mathrm{Tag}$ \\
\hline 38 & $\checkmark$ & Planung Step 2 & & & Don 04.12.14 & Don 04.12.14 & 1 Tag \\
\hline 39 & & Logistik Step 2 & & & Mon 12.01.15 & Mit 15.04.15 & 66 Tage \\
\hline 40 & & Angebotseinholung & & & Mon 12.01 .15 & Mon 02.03 .15 & 35 Tage \\
\hline 41 & & Bezug EWI & & & Mon 09.02.15 & Mit 04.03.15 & 18 Tage \\
\hline 42 & & 2 Fachpacker - Umzug Signatur A-D aus den Villen & & & Mon 09.02.15 & Fre 13.02.15 & 5 Tage \\
\hline 43 & & B 885 Judaistik & $614 \mathrm{~m}$ & Schwendenerstraße 27 & Die 24.02 .15 & Don 26.02 .15 & 3 Tage \\
\hline 44 & & B 867 Katholische Theologie & $910 \mathrm{~m}$ & Schwendenerstraße 31 & Fre 27.02.15 & Mit 04.03.15 & 4 Tage \\
\hline 45 & & Bezug Neubau ZKF & & & Mon 02.03.15 & Mit 15.04.15 & 31 Tage \\
\hline 46 & & Fertigstellung Neubau & & & Mon 02.03 .15 & Mon 02.03.15 & $1 \mathrm{Tag}$ \\
\hline 47 & & Umzug MA OVS 16 innerhalb & $6 \mathrm{MA}$ & & Mon 02.03 .15 & Mon 02.03 .15 & $1 \mathrm{Tag}$ \\
\hline 48 & & Umzug MA OVS $16>$ Van't Hoff 6 (Arrondierung) & $42 \mathrm{MA}$ & & Mon 02.03 .15 & Fre 06.03.15 & 5 Tage \\
\hline 49 & & Team I & & & Mon 02.03.15 & Die 14.04.15 & 30 Tage \\
\hline 50 & & B 802 Vorderasistische Archäologie & $500 \mathrm{~m}$ & Hüttenweg 7 & Mon 02.03 .15 & Die 03.03.15 & 2 Tage \\
\hline 51 & & EWI - Plus (B 870JA, B 870SI, B870KO, B816, B908, B726) & $2.000 \mathrm{~m}$ & Van't-Hoff 6 & Mit 04.03.15 & Die 10.03 .15 & 5 Tage \\
\hline 52 & & B 822 Klassische Archäologie & $1.180 \mathrm{~m}$ & Otto-v.-Simson-Str. 11 & Mit 11.03.15 & Die 17.03.15 & 5 Tage \\
\hline 53 & & B 871 Prähistorische Archäologie & $1.000 \mathrm{~m}$ & Altensteinstraße 15 & Mit 18.03.15 & Mon 23.03.15 & 4 Tage \\
\hline 54 & & B 896 Ägyptologie & $440 \mathrm{~m}$ & Altensteinstraße 33 & Die 24.03.15 & Mit 25.03.15 & 2 Tage \\
\hline 55 & & B 877 Iranistik & $545 \mathrm{~m}$ & Schwendenerstraße 17 & Don 26.03 .15 & Die 31.03.15 & 4 Tage \\
\hline 56 & & B 912 Turkologie & $180 \mathrm{~m}$ & Schwendenerstraße 33 & Mit 01.04.15 & Mit 01.04.15 & $1 \mathrm{Tag}$ \\
\hline 57 & & B 930 Sammlung Ehrlich & $280 \mathrm{~m}$ & Garystraße 39 & Mit 01.04.15 & Mit 01.04.15 & $1 \mathrm{Tag}$ \\
\hline 58 & & $\mathrm{BCP}$ & $2.700 \mathrm{~m}$ & Takustraße 3 & Don 02.04 .15 & Die 14.04.15 & 7 Tage \\
\hline 59 & & Team II & & & Mon 02.03.15 & Mit 15.04.15 & 31 Tage \\
\hline 60 & & Silberlaube Außenmagazin EL - Z & $3.000 \mathrm{~m}$ & Habelschwerdter Allee 45 & Mon 02.03 .15 & Fre 13.03.15 & 10 Tage \\
\hline 61 & & B 814 Physik & $1.850 \mathrm{~m}$ & Arnimallee 14 & Mon 16.03 .15 & Fre 20.03.15 & 5 Tage \\
\hline 62 & & B 879 Semitistik und Arabistik & $700 \mathrm{~m}$ & Altensteinstraße 34 & Mon 23.03.15 & Mit 25.03.15 & 3 Tage \\
\hline 63 & & B 812 Islamwissenschaften & $1.200 \mathrm{~m}$ & Altensteinstraße 40 & Don 26.03 .15 & Mon 30.03 .15 & 3 Tage \\
\hline
\end{tabular}


theken entfernt wurden. Diese Planung war auch deshalb von besonderer Relevanz, weil zeitgleich zum Auszug der Teilbibliotheken aus den Villenstandorten eine dritte Spedition die dort jeweils beheimateten Institutsangehörigen und deren Arbeitsmaterialien in den an die Campusbibliothek angrenzenden Gebäudeteil transportierte. Deshalb wurde es notwendig zu verhindern, dass ein Standort gleichzeitig von zwei Speditionen angefahren wurde. Gleichzeitig erschwerte dies aber auch die Anlieferung in die Campusbibliothek, weil eine Zufahrt in den ersten zwei Wochen des Umzugszeitraumes durch den Umzug der Fachbereichsmitarbeiter blockiert war und für den Bibliotheksumzug nicht zur Verfügung stand.

Wie schon während der Vorabintegrationen festgestellt, zeigte sich, dass der Zeitaufwand, um die angelieferten Kisten und Bücherwagen nach RVK in die Regale einzusortieren, um vieles größer war als das Tempo des Verpackens an den Quellstandorten. Daher wurde zu einem frühen Zeitpunkt während des Umzuges entschieden, die Mitglieder des Bibliotheksteams an der Aufgabe des Einstellens der Bücher am Zielstandort zu beteiligen. Dies bedeutete, dass über die Woche verteilt insgesamt 116 Personen daran beteiligt wurden, während die Speditionen ihre Mitarbeiter für das Einpacken an den Quellstandorten konzentrieren konnten. Dies führte wiederum dazu, dass der ursprüngliche Zeitplan, der sechs Wochen für den Transport und das Einstellen der Bücher veranschlagte, bedeutend schneller beendet werden konnte, und Kapazitäten für andere erforderliche Nacharbeiten, wie Stellrevision, Regalfahnenbeschriftung und Fehlerkorrekturen, frei wurden.

\section{Fazit}

Bereits zum „Bergfest“ stand fest, dass ein hervorragendes Team rund um den Umzug zusammengestellt worden war. Nicht nur, dass es keine größeren Probleme gab, wir hatten zu diesem Zeitpunkt - trotz widriger räumlicher Gegebenheiten - sogar schon einige Tage Planungsvorlauf.

Am 1. April 2015, gegen 16 Uhr, traf aus der Takustraße 3 die letzte LKW-Lieferung für den Neubau ein. Am 2. April gegen 10 Uhr wurde das letzte Buch im Neubau im Rahmen des großen Umzugs eingestellt. Vom 2. März 2015 bis zum 2. April 2015 hatten die beiden beauftragten Speditionen also 18475 Regalmeter oder 488241 Medieneinheiten transportiert und mit den Bibliotheksmitarbei- tern gemeinsam eingestellt. Geplant waren für diese Bestände sechs Wochen. Hinzu kommen 183452 Medien im Altbau, die bereits im Februar umgezogen worden waren. Die im Hintergrund laufenden Transporte der Bestände mit Magazinsignaturen (in den Magazinen) und der Bestände mit Altsignaturen (Magazine und Van't-Hoff-Str. 6) mitgezählt, wurden für die Campusbibliothek FU Berlin ca. eine Million Bände bzw. ca. 30 Kilometer Medien bewegt.

\section{Campusbibliothek}

Natur- Kultur- und Bildungswissenschaften Mathematik, Informatik und Psychologie Fabeckstraße 23-25 14195 Berlin Website: www.fu-berlin.de/campusbib Facebook: https://www.facebook.com/Campusbib.FUBerlin 